\title{
Urinary bladder cancer in Wegener's granulomatosis: is it more than cyclophosphamide?
}

\section{B Hellmich, I Kausch, C Doehn, D Jocham, K Holl-Ulrich, W L Gross}

\section{Development of tumours of the urinary tract is not necessarily caused by cyclophosphamide alone}

I this issue of the Annals Knight and coworkers from Sweden report some interesting data on the incidence of urinary bladder cancer in patients with Wegener's granulomatosis (WG) which, in contrast with previous reports from single or multicentre cohorts, are derived from a population based, nationwide inpatient register. ${ }^{1}$ The data of that study confirm the increased risk of bladder cancer in patients with WG after exposure to cyclophosphamide, but the results also indicate that there might be an increased risk for bladder cancer even before the onset of WG. ${ }^{1}$ Within a cohort of 1065 patients with WG eight patients had a diagnosis of bladder cancer before WG was diagnosed. Although not statistically significant, the increased incidence of bladder cancer seen before the onset of WG resulted in a relative risk of 2.1 (95\% confidence interval (CI) 0.6 to 3.6) compared with the expected prevalence of bladder cancer in Sweden.

These epidemiological data confirm an earlier report from our group from 1999, showing a relation of WG to tumours of the urinary tract for the first time in $1999 .{ }^{2}$ In that study we reported a significantly higher incidence of renal cell carcinoma in a single centre cohort of 477 patients with WG, resulting in an odds ratio of 8.73 (95\% CI 0.92 to 3.48 ; $\mathrm{p}<0.05){ }^{2}$ Interestingly, five out of seven patients developed WG and renal cell carcinoma simultaneously, whereas this temporal association was not found in any of the control patients with renal cell carcinoma and rheumatoid arthritis. ${ }^{2}$ This temporal association of the incidence of cancer and onset of WG is now confirmed by the study of Knight and coworkers. ${ }^{1}$ In the majority of their patients WG was diagnosed a relatively short time (median 1.5 years) after the onset of bladder cancer, ${ }^{1}$ which suggests that both events were somehow, if not causally, related. The odds ratio for simultaneous occurrence of cancer (all types) was 18 for patients with WG compared with control patients, ${ }^{2}$ whereas in a cohort of patients with different systemic vasculitides, a concurrence of solid tumours and vasculitis was found in 16 of 23 cases reviewed. ${ }^{3}$ Although the overall risk of developing malignant neoplasms in the Swedish cohort (OR 2.0, 95\% CI 1.7 to 2.5) ${ }^{4}$ and the German cohort (OR 1.79, 95\% CI 0.92 to 3.48$)^{2}$ was only modestly increased, patients with WG appear to be at an increased risk for certain other tumour subtypes. Beside renal cell and bladder carcinoma increased relative risks for squamous cell skin cancer (OR $7.3,95 \%$ CI 4.4 to 12.0 ), leukaemias (OR 5.7, 95\% CI 2.3 to 12), and malignant lymphomas (OR 4.2, 95\% CI 4.2 to 8.3 ) have been reported. ${ }^{4}$

\section{"Patients with WG seem to be at risk of developing several types of tumour'}

The mechanisms responsible for this association are unknown. Hypothetically, antineutrophil cytoplasmic antibodies (ANCA) may interact with proteinase 3 (PR3), the target antigen of ANCA in $W G,{ }^{5}$ expressed on the surface of the target organ (for example, bladder, kidney). However, studies of the presence of Wegener's autoantigen on epithelial cells have given conflicting results. Whereas King and coworkers did not detect expression of PR3 mRNA on renal epithelial cell lines, ${ }^{6}$ Mayet and coworkers reported the presence of PR3 mRNA and protein on distal tubular epithelial cells, glomerular epithelial cells in normal kidney tissues, $^{7}$ and pneumocytes (I and II). ${ }^{8}$ It was later shown that ANCA can induce signalling events in human renal epithelial cells by binding to PR3 expressed on the cell surface. $^{9}$ In the past year, it was demonstrated that the growth rate of tubular epithelial cells transduced with native PR3 was significantly enhanced to non-transduced epithelial cells. ${ }^{10}$ These recent data indicating a role of PR3 for differentiation and proliferation of not only haematopoietic cells but also epithelial cells may thus provide a theoretical link between Wegener's autoantigen and the development of solid tumours. While PR3 stimulates cell proliferation of myeloid cells by cleavage of the cyclin dependent kinase inhibitor p2l(wafl), ${ }^{11}$ signalling pathways involved in PR3 induced proliferation of non-myeloid cells have not yet been studied extensively. It is unclear whether PR3 expression is restricted to cells of certain organs and whether expression is found on cells from solid tumours including bladder cancer. Data for the presence of PR3 on tumour cells are contrasting. While expression of PR3 has been detected on mammary tumour cells, ${ }^{12}$ expression of PR3 was not found on the surface of tumour tissue from patients with WG in our previous study. ${ }^{2}$

As in most other malignancies the generation of bladder cancer is caused by the accumulation of different molecular changes. The expression of oncogenes such as ras, erbB-2, and EGF receptor; cell cycle genes such as pl5 or pl6; tumour suppressor genes such as $\mathrm{Rb}$ or p53; and DNA-repair genes is altered mostly by chromosomal aberration or mutation. Loss of heterozygosity of chromosome $9 p$ and $9 q$ has been shown to be a crucial event in the transition of normal urothelium to papillary transitional cell carcinoma, whereas p53 is primarily involved in the development of carcinoma in situ.

Various carcinogens and risk factors contribute to the generation of bladder carcinomas. Not much is known about genetic bladder cancer disposition in patients with Wegener's granulomatosis. For the rare carcinosarcoma of the bladder, heterozygosity and microsatellite marker studies have recently indicated a monoclonal origin of WG and the bladder tumour, including allelic loss of chromosome 9 p. $^{13}$

Owing to the increased risk of bladder cancer, a routine urological examination should be performed in patients after exposure to cyclophosphamide and especially in patients with concomitant micro- or macrohaematuria. For the early diagnosis of bladder cancer, cystoscopy and urine cytology is still the preferred method. In addition, several molecular tests which are based on urine analysis have yielded promising results and are beginning to be established in clinical diagnosis and follow up of bladder cancer. These molecular tests mostly detect certain antigens or 
degradation products which are highly or specifically expressed in bladder tumours, such as for instance bladder tumour antigen and nuclear matrix protein 22, or detect certain common chromosomal aberrations.

\section{"New tests are becoming available} for the detection of bladder cancer"

Most bladder neoplasms are transitional cell carcinomas and about $80 \%$ of them are superficial tumours that do not reach the muscular layer of the bladder wall. Superficial tumours are usually treated by transurethral resection with and without adjuvant intravesical chemo- or immunotherapy, and muscle-invasive tumours normally indicate the need for radical cystectomy or radiotherapy. Most superficial tumours can be cured by transurethral resection, but $20-30 \%$ will undergo local tumour progression into muscle-invasive disease. The 5 year progression-free survival after radical cystectomy or radiotherapy in patients with muscleinvasive tumours is $50-60 \%$. Thus, early detection of bladder malignancies is important.

\section{HAEMORRHAGIC CYSTITIS, BLADDER CANCER, AND THE ROLE OF CYCLOPHOSPHAMIDE}

Beside the risk of developing haemorrhagic cystitis associated with WG, a large body of evidence suggests that administration of cyclophosphamide is linked to an increased risk for bladder cancer in patients with WG. ${ }^{44-16}$ Overall, there is a nine- to 45 -fold increased risk of bladder cancer in patients treated with cyclophosphamide. ${ }^{17-19}$ In their large cohort of patients with WG, Knight and coworkers reported a 31-fold relative risk and a cumulative risk of $3 \%$ at 10 years and $10 \%$ at 15 years after treatment with cyclophosphamide. ${ }^{4}$ In other studies with median times of follow up varying from 7 to 20 years, cumulative incidences of bladder cancer of $<1 \%, 2.7 \%$, 3\%, and $5 \%$ have been reported. ${ }^{14-16} 20$ Evidence from these studies suggests that the development of bladder cancer in patients with WG is related to both the duration of cyclophosphamide treatment and the cumulative doses used. It appears that the relative risk is increased for cumulative cyclophosphamide doses over $25 \mathrm{~g}$ or an exposure of more than 12 months $^{4}$ and particularly high for cumulative cyclophosphamide doses over $100 \mathrm{~g}$ or an exposure of more than 2.5 years. ${ }^{15}$

The absolute number of confirmed cases of bladder cancer in the different WG cohorts is low and thus comparisons have to be made with caution. However, there is considerable evidence that the risk of bladder cancer in patients with WG is clearly linked to previous episodes of haemorrhagic cystitis, for which cyclophosphamide is also a strong risk factor. In a cohort of 145 patients with WG observed at the NIH, all seven patients with bladder cancer had at least one episode of nonglomerular haematuria. ${ }^{15}$ In contrast, none of the 72 patients in that cohort who did not have a documented episode of non-glomerular haematuria developed bladder cancer. ${ }^{15}$ In the study by Knight et al only one patient had an episode of haematuria leading to cystoscopy before the diagnosis of bladder cancer. ${ }^{1}$ However, in contrast with other monocentric cohorts these patients were not systematically followed up as outpatients and thus minor intermittent episodes of haemorrhagic cystitis might have been missed. Although the design of that study was not appropriate for detecting haemorrhagic cystitis systematically, haemorrhagic cystitis should still be considered as a risk factor for bladder cancer in $W G$, given the strong association shown in previous studies. ${ }^{15} 1620$

\section{ROLE OF MESNA FOR UROPROTECTION IN CYCLOPHOSPHAMIDE TREATED PATIENTS}

In view of the above mentioned association of bladder cancer with previous episodes of haemorrhagic cystitis, measures aimed at reducing the incidence of haemorrhagic cystitis in patients receiving cyclophosphamide may also reduce the risk for bladder cancer. After oral or intravenous administration, cyclophosphamide is first metabolised in the liver to hydroxycyclophosphamide and later by target cells to pyrophosphamide mustard and acrolein. ${ }^{21}$ Pyrophosphamide mustard acts cytotoxically by forming a covalent linkage with nucleic acids and thus mediates the immunosuppressive effects. In contrast, acrolein is not an immunosuppressant, but is excreted by the kidney and causes toxic effects on the bladder epithelium and is thus responsible for the urothelial damage caused by cyclophosphamide. ${ }^{22}$ In a prospective randomised study involving bone marrow transplant recipients after administration of cyclophosphamide, mesna (2-mercaptoethane sulphonate sodium) significantly reduced the incidence of haemorrhagic cystitis compared with a control group treated with forced diuresis. ${ }^{23}$ Whether mesna is uroprotective in cyclophosphamide treated patients with WG is still a matter of debate, ${ }^{24}$ but growing evidence suggests that mesna can in fact reduce the incidence of haemorrhagic cystitis and bladder cancer in WG.

Although the study by Knight and coworkers in this issue of the Annals makes a very important contribution to information about the prevalence of bladder cancer in $\mathrm{WG}^{1}{ }^{1}$ information about the usefulness of mesna in that paper is very limited as (a) only three patients in the whole cohort had received mesna; and $(b)$ data on intermittent episodes of haemorrhagic cystitis in that cohort from an inpatient registry are probably insufficient, as outlined above. We therefore feel that the authors' hypotheses suggesting that bladder cancer is unrelated to haemorrhagic cystitis and that mesna may not be cancer protective are premature.

\section{"Mesna may reduce the incidence of haemorrhagic cystitis and blad- der cancer in WG"}

Because prospective comparative studies of mesna and placebo have demonstrated uroprotection by mesna in malignant disease, ${ }^{23}$ but have not yet been performed in WG, estimates of the benefit of mesna in WG can only be obtained by comparison of carefully observed cohorts which either did or did not use mesna. Patients in the large cohort of patients with WG observed at the NIH did not receive mesna along with cyclophosphamide treatment. ${ }^{14}{ }^{15}$ In that cohort, 68/158 (43\%) patients and, after extended follow up, 42/145 (29\%) patients developed haemorrhagic cystitis due to cyclophosphamide treatment. ${ }^{14}{ }^{15}$ In contrast, in patients receiving mesna together with cyclophosphamide in the German WG cohort reported by Reinhold-Keller and coworkers, only 17/142 (12\%) patients had cyclophosphamide induced cystitis. ${ }^{16}$ Comparison of both cohorts shows a relative risk for the occurrence of haemorrhagic cystitis of $0.41 \quad$ (95\% CI 0.25 to 0.69 ) without administration of mesna compared with only 0.28 (95\% CI 0.17 to 0.45 ) with administration of mesna. ${ }^{25}$ The incidence of haemorrhagic cystitis in the cohort given mesna was significantly lower than the incidences reported in the two NIH studies where no mesna was administered $(\mathrm{p}=$ 0.00001 and $p=0.0004$, respectively). ${ }^{25}$

Furthermore, the incidence of bladder cancer was significantly lower in the mesna treated cohort (1/155 patients) than in the NIH cohort not treated with mesna $(7 / 158$ patients; $p=0.034) .{ }^{25}$ Results of several studies have shown that the occurrence of bladder cancer increases with the duration of follow 
up. ${ }^{15}$ For example, in the study by Knight and coworkers the incidence of bladder cancer was only $2 \%$ after 10 years, but it was $10 \%$ after 16 years of follow up. ${ }^{1}$ Given this association between duration of follow up and the incidence of bladder cancer, the difference between the NIH cohort and the German cohort in favour of a benefit of mesna might have been even greater as the observation period in the German study was longer (2144 patient-years) than in the two NIH studies (1229 and 1331 patient-years, respectively). ${ }^{14-16}$ Notably, further extension of the follow up period in the mesna treated German cohort from a median of 7 years by an additional 39 months did not show any new cases of bladder, ${ }^{25}$ suggesting a protective effect of mesna.

With the exception of rarely occurring allergic reaction, which may mimic a vasculitic rash, mesna is well tolerated. ${ }^{26}$ We therefore propose the prophylactic use of mesna for prevention of bladder toxicity in patients with WG receiving cyclophosphamide. Alternative strategies to reduce the urotoxic effect of cyclophosphamide include forced diuresis and efforts to reduce the exposure to cyclophosphamide by pulse administration or use of alternative agents like methotrexate or mycophenolate mofetil, if clinically indicated.

In summary, the epidemiological data reported by Knight et al in the present issue of the Annals confirm our previous clinical observation, showing that development of tumours of the urinary tract is not necessarily caused by cyclophosphamide alone, but appears to some extent to be related to the underlying autoimmune disease itself. In view of this, we propose a systemic investigation of patients with newly diagnosed WG in order to exclude concomitant malignant neoplasms. Further studies are needed to clarify whether PR3, Wegener's autoantigen, may be the missing link between autoimmunity and uncontrolled cell proliferation in patients with WG and malignant disease.

Ann Rheum Dis 2004;63:1 183-1185.

doi: 10.1136/ard.2004.023937

\section{Authors' affiliations}

B Hellmich, W L Gross, Poliklinik für Rheumatologie, Universitätsklinikum Schleswig-Holstein, Campus Lübeck, Ratzeburger Allee 160, 23538 Lübeck, Germany

I Kausch, C Doehn, D Jocham, Klinik und Poliklinik für Urologie, Universitätsklinikum Schleswig-Holstein, Campus Lübeck, Ratzeburger Allee 160, 23538 Lübeck, Germany

K Holl-Ulrich, Institut für Pathologie, Universitätsklinikum Schleswig-Holstein, Campus Lübeck, Ratzeburger Allee 160, 23538 Lübeck, Germany

Correspondence to: $\operatorname{Dr} B$ Hellmich; bernhard.helllmich@rheuma.uni-luebeck.de

\section{REFERENCES}

1 Knight A, Askling J, Granath F, Sparen P, Ekborin A. Urinary bladder cancer in Wegener's granulomatosis: risks and relation to cyclophosphamide. Ann Rheum Dis 2004:63:1307-11

2 Tatsis E, Reinhold-Keller E, Steindorf K, Feller AC Gross WL. Wegener's granulomatosis associated with renal cell carcinoma. Arthritis Rheum 1999;42:751-6

3 Kurzrock R, Cohen P. Clinical manifestations of vasculitis in patients with solid tumors. Arch Intern Med 1994; 154:334-42.

4 Knight A, Askling J, Ekbom A. Cancer incidence in a population-based cohort of patients with Wegener's granulomatosis. Int $J$ Cancer 2002; 100:82-5.

5 Lüdemann JBU, Gross W. Anti-neutrophil cyłoplasmic antibodies in Wegener's granulomatosis recognize an elastinolytic enzyme. J Exp Med 1990;171:357-62.

6 King WJ, Adu D, Daha MR, Brooks CJ, Radford DJ, Pall AA, et al. Endothelial cells and renal epithelial cells do not express the Wegener's autoantigen, proteinase 3. Clin Exp Immunol 1995; 102:98-105.

7 Schwarting A, Hagen D, Odenthal M, Brockmann H, Dienes HP, Wandel $E$, et al. Proteinase-3 mRNA expressed by glomerular epithelial cells correlates with crescent formation in Wegener's granulomatosis. Kidney Int 2000;57:2412-22.

8 Brockmann H, Schwarting A, Kriegsmann J, Petrow P, Gaumann A, Muller KM, et al. Proteinase- 3 as the major autoantigen of c-ANCA is strongly expressed in lung tissue of patients with Wegener's granulomatosis. Arthritis Res 2002;4(3):220-5. Epub 2002 Mar 27

9 Hattar K, Grandel U, Bickenbach A, Schwarting A, Mayet WJ, Bux J, et al. Interaction of antibodies to proteinase 3 (classic antineutrophil cytoplasmic antibody) with human renal tubular epithelial cells: impact on signaling events and inflammatory mediator generation. $\mathrm{J}$ Immunol 2002;168:3057-64.

10 Relle M, Mayet WJ, Strand D, Brenner W, Galle PR, Schwarting A. Proteinase
$3 /$ myeloblastin as a growth factor in human kidney cells. J Nephrol 2003;16:831-40.

11 Witko-Sarsat V, Canteloup S, Durant S,

Desdouets $C$, Chabernaud R, Lemarchand $P$, et al. Cleavage of $\mathrm{p} 21$ waf1 by proteinase- 3 , a myeloid-specific serine protease, potentiates cell proliferation. J Biol Chem 2002;277:47338-47. Epub 2002 Sep 26.

12 Horman S, Fokan D, Galand P. MCF-7 mammary tumour cells express the myeloid cell differentiation controlling factor, serine protease 3/myeloblastin. Cell Prolif 2000;33:331-40.

13 Mukhopadhyay S, Shrimpton $A E$, Jones LA, Nsouli IS, Abraham NZ Jr. Carcinosarcoma of the urinary bladder following cyclophosphamide therapy: evidence for monoclonal origin and chromosome 9p allelic loss. Arch Pathol Lab Med 2004; 128: 8 -11.

14 Hoffman G, Kerr G, Leavitt R, Hallahan CW Lebovics RS, Travis WD, et al. Wegener's granulomatosis: an analysis of 158 patients. Ann Intern Med 1992; 1 16:488-99.

15 Talar-Williams C, Hijazi YM, Walther MM, Linehan WM, Hallahan CW, Lubensky I, et al. Cyclophosphamide-induced cystitis and bladder cancer in patients with Wegener granulomatosis. Ann Intern Med 1996;124:477-84.

16 Reinhold-Keller E, Beuge N, Latza U, de Groot K, Rudert $\mathrm{H}$, Nolle B, et al. An interdisciplinary approach to the care of patients with Wegener's granulomatosis: long-term outcome in 155 patients. Arthritis Rheum 2000:43:1021-32. Erratum in: Arthritis Rheum 2000;43:2379.

17 Fairchild W, Spence C, Solomon H, Gangai M. The incidence of bladder cancer after cyclophosphamide therapy. J Urol 1979;122:163-4.

18 Ansell I, Castro J. Carcinoma of the bladder complicating cyclophosphamide therapy. Br J Urol 1975;47:413-18.

19 Stillwell TJ, Benson RC Jr. Cyclophosphamideinduced hemorrhagic cystitis. A review of 100 patients. Cancer 1988;61:451-7.

20 Stillwell TJ, Benson RC Jr, DeRemee RA, McDonald TJ, Weiland LH. Cyclophosphamideinduced bladder toxicity in Wegener's granulomatosis. Arthritis Rheum 1988;31:465-70.

21 Coggins P, Ravdin R, Eisman S. Clinical pharmacology and evaluation of Cytoxan (cyclophosphamide). Cancer Chemother Rep 1959;3:9-11.

22 Cox P. Cyclophosphamide cystitis: identification of acrolein as the causative agent. Biochem Pharmacol 1979:28:2045-9.

23 Hows JM, Mehta A, Ward L, Woods K, Perez R, Gordon MY, et al. Comparison of mesna with forced diuresis to prevent cyclophosphamide induced haemorrhagic cystitis in marrow transplantation: a prospective randomised study. Br J Cancer 1984;50:753-6.

24 Sneller MC. Cystitis, bladder cancer, and myelodysplasia in patients with Wegener's granulomatosis: comment on the article by Reinhold-Keller et al [letter]. Arthritis Rheum 2000;43:2853-4.

25 Reinhold Keller E, de Groot K, Gross W. Reply to: Cystitis, bladder cancer, and myelodysplasia in patients with Wegener's granulomatosis [letter]. Arthritis Rheum 2000;43:2854

26 Reinhold-Keller E, Mohr J, Christophers E, Nordmann K, Gross WL. Mesna side effects which imitate vasculitis. Clin Investig 1992;70:698-704 